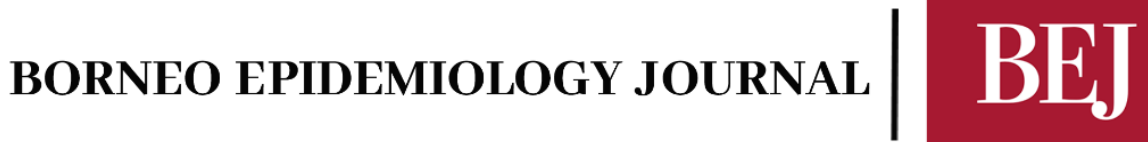

\section{Public Health Informatics in Global Health Surveillance: A Review}

Puteri Nureylia Amir, Mohd Fazeli Bin Sazali*, Loganathan Salvaraji, Nafsah Dulajis, Syed Sharizman Syed Abdul Rahim, Richard Avoi

\begin{abstract}
Background: Surveillance is the backbone for effective public health practice. Traditionally, surveillance system relies on the collection of information regarding health-related events through healthcare facilities, disease notification system from the physician, syndromic notification networks, selected sentinel healthcare facilities, or by event-based data. However, there are several limitations in using conventional surveillance.
\end{abstract}

Methods: With the advancement of technology and computer science, overcoming those limitations and complementing the traditional method has been recommended. Three leading emerging technologies are applied in public health surveillance: the internet of things, artificial intelligence, and blockchain.

Results: Application of informatics in public health surveillance could raise several issues including accessibility and affordability of innovations; public health informatics' experts, law, and regulation to protect patients' information; social and ethical considerations, norms, and standards of implementing new technologies; data ownership; privacy and sharing of information; biosecurity; biosafety; and cybersecurity.

Conclusion: This article aimed to review several applications of informatics system in public health surveillance practice and its several issues related to the use of technology. Several applications of informatics could be useful for incoming challenges in public health. However, application of informatics can pose significant issues and must be taken into consideration in public health practice.

Keywords: Public health informatics, Surveillance, Event-based surveillance, Web-based surveillance.

*Correspondence Email: drmohdfazeli@ gmail.com

Department of Public Health Medicine, Faculty of Medicine and Health Science, Universiti Malaysia Sabah, Jalan UMS, 8840, Kota Kinabalu, Sabah, Malaysia

Received: 08/11/2021

Accepted: 10/12/2021 


\section{Introduction}

Public health surveillance is the backbone for effective public health practice. The first concept of surveillance was first introduced in 460 B.C. by Hippocrates when he introduced the term endemic. It provided the concept of collecting data regarding the place, environment and people's behavior (Choi B.C.K., 2012). Afterwards, the component of surveillance that includes an ongoing and systematic data collection, analysis, interpretation and timely dissemination of health-related data had been applied and gone through historical evolvement until what is presented today (Choi B.C.K., 2012 \& CDC, 2014).

Informatics is generally can be used by other fields such as business, security, social science, and others. It can also be applied to public health. It can be used to enable effective monitoring and surveillance, to support improved decision-making, promote health, preventing disease and injuries, and to improve the health of the population (Aziz, H.A., 2016 \& PHII, 2020). In the scope of public health, public health informatics is defined as a systematic application of information using computer science and technology into public health practice $(C D C, 2014)$. The public health practice that utilizes informatics includes surveillance, prevention, preparedness, health promotion, research and learning (Aziz, H.A., 2016 \& CDC, 2014). As modern days has approached, computer technology has becoming an essential tool to enhance the use of public health surveillance system nowadays (Aziz, H.A., 2016 \& McNabb, S.J.N. et al., 2017). Even though informatics has been widely used in many industries, its application in public health was limited in literature. This article aimed to review several applications of informatics system in public health surveillance practice and its several issues related to the use of technology.

\section{Application of Public Health Informatics in Surveillance}

Traditionally, surveillance system relies on the collection of information regarding healthrelated events through healthcare facilities, disease notification system from the physician, syndromic notification networks, selected sentinel healthcare facilities, or by event-based data. It is one of the core functions in public health as it can provide an early warning system for disease outbreaks or disasters, served as an advocacy tool for informing public health policy and strategies, evaluate the effectiveness of an intervention, and set the priority of the intervention in a cost-effective manner (WHO, 2020).

However, there are several limitations in using conventional surveillance. Underreporting, time-consuming, delay in reporting and subsequent public health intervention, expensive, poor integration, and lack of representativeness are among various challenges in public health surveillance (Mandyata, C.B. et al., 2017 \& CDC, 2012). With the advancement of technology and computer science, the technology has been increasingly recommended in overcoming those challenges and serves as complementary method to the traditional way of surveillance activity (Jayatilleke, K., 2020).

\section{Emergence of New Technology in Surveillance}

With the increasing amount of public health data, together with the growing importance of public health informatics, it is undeniable that public health officials need to better understand on current trend of technology and its impact. A paradigm shift is critical to be adopted by public health practitioner to integrate emerging technology innovation in public health. For greater impact of utilization of these technologies, public health practitioner needs to have 
good relationship and collaboration with the experts in computational science. Greater understanding in technology in health can guide in future training and continuous education in public health program. Previous literature review identified three main emerging technologies that are applied in public health surveillance activity, namely Internet of Things, Artificial Intelligence, and Blockchain (Huang, J., 2019).

\section{i. Internet of Things}

Internet of Things (IoT) refers to the concept where the systems are interrelated and internetenabled connections that are able to collect and transfer data between objects without human intervention (What is IoT?). It is becoming an emerging paradigm that allows wireless communication between electronic devices and sensors to facilitate human daily activities. It provides innovative solution to various challenges, especially in public health surveillance. There is big potential of IoT considering that currently there are 10 billion IoT devices in 2020 and it was projected to increase to 25 billion in 2025, and the data generated is expected to reach 3.1 ZB (zettabytes) by year 2025 (45 Fascinating IoT Statistics for 2021). IoT has been emergently used in healthcare industry. For example, wearable sensors can be used to identify individuals with COVID-19 infection by remotely monitoring clinical parameters (oxygen, temperature, heart rate, and blood pressure) and subsequently can be integrated with the special hardware and smartphone application devices (Mukhtar, H. et al., 2021).

\section{ii. Artificial Intelligence}

Artificial intelligence (AI) is generally referring to ability of digital technology to perform task that is typically associated with intelligent being. AI can be defined as science and engineering of making intelligent machines, especially intelligent computer programs. It is related to the similar task of using computers to understand human intelligence (Mccarthy J., 2007). In the simpler form, AI is a computer science approach to create intelligent machines. One of important subset of AI is machine learning. Machine learning refers to the ability of the machine to learn from the past data without being explicitly programmed. Application of $\mathrm{AI}$ and machine learning enable the decision making and action become faster. It has been widely applied in public health especially in identification of emerging threats (e.g., COVID19), enable detailed and up-to date understanding of population disease and risk factors distribution, disease incidence forecasting, guiding for targeted approach in intervention, assessment of effectiveness of intervention, as well as advocacy tool for policy changes (Zeng, D., 2021). Data aggregation system, such as Global Public Health Intelligence Network (GPHIN) and Program for Monitoring Emerging Diseases (ProMED) are among the most common example of application of AI and machine learning to crowdsource, process, and filter online data (Budd, J. et al., 2020).

\section{iii. Blockchain}

Blockchain is basically referring to the decentralized, publicly distributed digital ledgers that consist of records that is called blocks to record transaction across multiple computers (How IoT, AI, and blockchain will revolutionize business, 2018). One of the special features is that transactional record cannot be retrospectively altered, without the alteration of other subsequent blocks. The main advantage of applying this blockchain is the process is faster, visible in real-time manner, and reduced operational cost. In healthcare context, blockchain has been used to promote patient-centered care by sharing patient data for remote monitoring and management (Bhattacharya, S. et al., 2019). In public health context, blockchain is being 
used in terms of sharing of genomic data (Friedrich, M.J., 2019). One of example of usage of blockchain is during E. coli outbreak in Denmark which it involved the sharing of DNA data of the bacteria between Denmark, German, and Spanish scientists to identify the source of epidemic (Bellod, C.J.L., 2018). The importance of blockchain, especially in global health can no longer be denied, as we learnt from Nipah virus epidemic and influenza virus pandemic (How One Man Saved his Country, 2017).

\section{Example of Technology Application in Surveillance}

\section{i. Event-Based Surveillance (EBS)}

Different from the indicator-based surveillance that systematically collects information from the official reports, EBS is a surveillance method that collects and analyses the unstructured data from various source of information such as news reporting, social media, and internetbased searches (Balajee, S.A. et al., 2021). Typically, disease reporting is conducted through disease notification by physician. This requires the patient to self-aware their need to see the physician upon experiencing the symptoms. In COVID-19 infection, one fifth of the patients are asymptomatic (Kim, G.U. et al., 2020) and among those who are symptomatic, only $46.8 \%$ of them seek medical care (Meng, H. et al., 2016). This might cause under-reporting of disease and missing the cases in which healthcare is not sought. Therefore, identifying undetected cases could be enhanced using digital technology to elucidate the magnitude and characteristics of the outbreak and reduce transmission of the disease.

It was documented that majority of initial data $(60 \%)$ that were collected for surveillance activities were originated from unofficial informal sources, including those obtained from social media, internet, and mainstream media (WHO, Epidemic Intelligence, 2020). Therefore, digital technology is one of the important tools to improve the surveillance system and has been used to utilise this informal information for rapid identification of events with potential epidemic. This is in particularly important especially when there is an ongoing global pandemic of COVID-19 which recorded 166 million cases with more than 3 million deaths, by May 2021 (WHO Coronavirus (COVID-19) Dashboard, 2021).

One of the most important event-based surveillance systems using this digital approach is the Global Public Health Intelligence Network (GPHIN). GPHIN is a network that systematically scans all the multilingual informal sources such as online news sites, social networks, web searches, participatory longitudinal community cohorts, and other data aggregation systems (eg. ProMED-mail) solves unusual events and rumors by using natural language processing and machine learning processes. Typically, there were approximately 7000 articles and news feeds into GPHIN for analysis, and there were almost 3000 feeds contain duplication or irrelevant data. All the data will undergo data processing and analyst assessment that consist of several activities such as deduplication, metadata, categorization, translation, and relevancy scoring. In the final analysis, there was only 5-10 feeds were included in the daily report (Blench, M., 2008). GPHIN has been used in collaboration with WHO since 1997 and it has been used as part of the WHO's Global Outbreak Alert and Response Network (GOARN). Utilisation of GPHIN is able to meet the requirement of International Health Regulation 2005 core capacity, to provide early warning system to all WHO country members, enable reporting of unusual events, and for dissemination of information to public health officials all over the world in time-sensitive manner (Kamradt, S.A., 2019). 
Use of informal source of information provides several advantages over the formal source of information. In the evaluation of Avian influenza surveillance, informal information source reported cases 2.3 days earlier than WHO reports (Brownstein, J.S., 2008). Meanwhile, in another study found that among 111 outbreaks reported, formal source of information has delay in reporting by 1.26 day $(\mathrm{p}=0.002)$ compared to informal information (Bahk, C.Y. et al., 2015). This shows that the unstructured information might be valuable in surveillance system. However, the use of informal information needs to be conducted with caution, as previous study found that even though it was found to be superior in term of timeliness, but it has problem with sensitivity (only 44\%) (Brownstein, J.S., 2008). This problem was arising because most of systems that used informal source of information accumulate large amount of information in a wide variety of illnesses, leading to difficulty in extracting important information from the data (Magid, A. et al., 2018). This limitation can be overcoming by data processing and analyst assessment to filter out irrelevant and duplicated information (Blench, M., 2008).

The other commonly used event-based system including ProMED-mail (ProMEDmail), which is using sophisticated data processing and text mining to filter and classify the emails, articles, and news in the internet. The system is a largest publicly accessible system that is mainly conducting the infectious disease outbreak worldwide. The system is adopting one health approach, where the information obtained by the system will be filtered and analyzed by a multidisciplinary team representing various fields such as epidemiology, virology, parasitology, veterinary, and plant disease. The contribution of ProMED in changing the global surveillance landscape has been recognized as among the first system that alerted global community regarding infectious disease outbreak that have pandemic potential such as Severe Acute Respiratory Syndrome (SARS) in Guandong, China in 2003, Middle East Respiratory Syndrome Coronavirus (MERS-CoV) in Saudi Arabia in 2012, and numerous additional outbreaks of Zika, Nipah, Ebola, and other terrifying diseases (Whitelaw, S., 2020). Ongoing COVID-19 pandemic also has been successfully reported by ProMED with 109 entries reported between February 29, 2000 and January 22, 2020 (Bonilla, A.D.K. et al., 2020). Other widely used event-based system that provide useful tool in surveillance including Argus (Autonomous Real-Time Ground Ubiquitous Surveillance Imaging System) (ARGUS-IS), HealthMap (Flu \& Ebola Map, Virus), EpiSPIDER (Semantic Processing and Integration of Distributed Electronic Resources for Epidemics [and disasters]), and BioCaster. All of these different systems might have some overlap in their function, but those systems intended to be complementary to the conventional type of surveillance, as they are differs in term of their way of data collection, source of information, language used, degree of automation, data analysis and interpretation, and how the data was visualized for information dissemination (Magid, A. et al., 2018).

\section{ii. Online Real Time Surveillance}

\section{a. Symptom Reporting}

Google Flu Trend (GFT), Flu near you, and Influenza Net are among the well-known online surveillance system that has been developed to support the conventional surveillance. GFT was one of the first web-based systems for tracking influenza activity in real time. The initial motivation of GFT development was to be able to predict the influenza disease activity early and respond immediately to reduce the impact of influenza infection. The GFT was proposed to supplement the surveillance system through prediction of influenza outbreak with 1-2 weeks earlier than the official report by United States Centre for Disease Control and 
Prevention (CDC). The estimation is based on the study that the number of people searching for influenza related information in website is strongly correlated with the number of people who having influenza like illness (ILI) symptoms and seeking care in hospital (Klembczyk, J.J. et al., 2016). Early Google publication on the GFT ability to predict influenza infection were 97\% accurate compared with CDC data (Ginsberg, J. et al., 2009).

The GFT has been performed well since its development in 2008 and was adopted by 29 countries. However, subsequent report of GFT has been documented its questionable accuracy. Study conducted in eight Latin America countries found that GFT are inaccurate in predicting influenza activity especially in tropical countries with irregular influenza seasonality, but more accurate in countries with more regular influenza seasonality (Pollett, S. et al., 2017). The GFT system was found to be over-estimating the influenza incidence, where in one interval from 2012 to 2013 during flu season, it predicted influenza incidence was two times higher than the actual emergency department visit for influenza related illness (Butler, D., 2013). Inaccuracy in estimation by GFT was assumed to be confounded by the impact of extensive media coverage and changes in health seeking behaviour that was triggered by the declaration of a public-health emergency by local authority to anticipated rise in influenza infection (Butler, D., 2013). Even though the web based real time surveillance has been limited its usability due to lack of evidence of its accuracy, a number of more advanced linear and nonlinear techniques to ILI modelling have been suggested, which shows promising results (Lampos, V. et al., 2015).

\section{iii. Wearables and sensors}

Other than using the web-based surveillance, the other approach is using several tools that is decentralized and connected with online system. Case identification using sensors, including thermal imaging camera and infrared sensors, are widely used with the basis to identify individuals with febrile illness. The instrument is commonly deployed in the areas with high population mobility, such as in airports, shopping malls, and public buildings. The data that is captured from the sensors are then will be connected to a centralized system and geospatially tracked to identify areas with emerging cluster of infection where the targeted public health intervention can be conducted, as being conducted in Singapore (Singapore's COVID-19). Meanwhile, John Hopkins researcher has launched a mobile application to study the geographical areas with COVID-19 infection as well as using some prediction modelling to predict the disease activity in an area using machine learning and spatial analytics (Johns Hopkins Team).

In addition, to cover the remote areas with large population density as well as poor internet connectivity, drones or unmanned aerial vehicles (UAV) might be useful. The tool is used to collect the data similar with the thermal sensor. The tool also can be used to monitor people's adherence to social distancing and application of face mask (Kumar, A. et al., 2021). However, utilization of thermal sensors is not without its limitation. There are large proportions of COVID-19 patients are asymptomatic (20\% asymptomatic patients in South Korea and 35.5\% in Petaling District, Selangor, Malaysia) (Kim, G.U. et al., 2020 \& Rama K.S. et al., 2021), use of antipyretics, people's movement variability, and practical issues (variability of body temperature in response to climatic changes, building temperature, device accuracy) might potentially affect the reliability of thermal sensors (Wright, W.F. et al., 2021). Utilization of these tools, if it being considered should be linked with the ongoing public health surveillance system, so that it can be monitored, followed by appropriate intervention including isolation of cases and quarantine of contacts. 


\section{ISSUES AND CHALLENGES RELATED TO PUBLIC HEALTH INFORMATICS USE IN SURVEILLANCE}

Advancement in innovations and technologies will create the pace of change and new opportunities, inversely, possibly aggravate existing divisions and disparities. Big data and automation pose the several risks to the world (Davis, E., 2017).

\section{Accessibility and Affordability}

There are deliberate concerns that innovations will increase expenses in healthcare system. A syndrome surveillance system was established by Boston Public Health Commission (BPHC), in collaboration with the Centre for Disease Control and Prevention (CDC), and the Massachusetts Department of Public Health. The total cost of developing system was nearly $\$ 450,000$ (Kirkwood A. et al., 2007).

Purchasing innovations may be costly and return of investment will take time. Especially among low- and middle-income countries where there is a lack of mechanism to systematically collect, analyze and use these data. Lack of sufficient and reliable data collecting health information system has been reported as one of the major barriers for effective public health action at country level (Mathers, C.D. et al., 2005). In addition, due to lack of comprehensive and quality local data, major donors are in doubt to provide financial support for innovative solutions, such as computer-based modelling (Butler, D., 2017). Resource-limited countries such as Africa, Asia, and other parts of the world struggling to build a healthcare facility acquired with information system. Hence, these countries constrain for adequate domestic disease detection or response capabilities (Hitchcock, P. et al., 2007).

Although International Health Regulation (IHR) 2005 provides a legal framework for global surveillance and response to communicable disease, yet fund for the program was not highlighted. At the country level, strategy plan was not available to raise the financial resources to implement preventive measure as mentioned in the revised IHR 2005. The Global Outbreak Alert and Response Network's (GOARN) operating budget seems to be not part of WHO's core budget (Baker, M.G. et al., 2006).

Not all health information system is costly to assess the full impact of information systems and informatics interventions. Several authors relate to the impact of systems in the quality of care. A study to evaluate the electronic health records (EHR) and investigates on the quality and efficiency of patients presenting to emergency departments. The system proves to be associated with salutary outcomes and prolongation of emergency length of stay (Connelly, D.P. et al., 2012). In Louisiana, the public health informatics team implemented an integrated electronic medical record (EMR) for HIV/AIDS. This system successfully reduces critical opportunities to intervene with defaulter, vast number of information collected solely for public health purposes (Herwehe, J. et al., 2012).

\section{Workforce}

Since not many experts in the field of public health informatics, it is impossible to effectively implement and adopt workforce right person for right job. New innovations and technologies have essential workforce implications. Recognizing the nature of the workforce of the future will be different from the workforce of present. Some job tasks by human may be negatively affected if certain considerations are not reviewed. Probably some working opportunity will 
be diminished while others will be transformed. Recent study on Public Health Information course available in the world highlighted the average number of credits and the study costs required were much higher in private as compared to public institutions. Hence, the study proposes the need for online contextual and cost-effective PHI training programs exists to occupy the increasing needs of professionals to improve public health in their respective countries (Joshi, A. et al., 2012).

To manage new data and new technologies, public health professional should lead best practice of advancement of technology. Integrated committee should be established (e.g., government, academia and not for profit public health institutions) to ensure that the technologies commonly developed at academic institutions make it to primary health units and public health organizations able to capture reliable data for improve of public health measures (McNabb, S.J.N. et al., 2017).

The role of policymakers and other stakeholders, such as professional organizations, will need to pre-plan about the consequences and implications for education and training. The evolvement of teaching and knowledge has to be updated for those entering the workforce. Continuous retrain the existing workforce to align with evolving technologies and labor trends should be intensified.

\section{Regulation}

Regulation ensures patients are protected from emerging technologies. Especially those that enter the market before reliable evidence is available to ensure this are the case. Data protection laws differ from countries; require designated organization of where data is stored. European countries such as Spain, France or Italy denied storage of patient data in the cloud. Nevertheless, some cloud providers allow obligatory data storage in a specific geographic location (Health Management).

We need to investigate the way evidence is collected and evaluated. The major challenge of data quality that comes from vast number of unchecked data can be lessened somewhat by innovations in PHI. Automatic systems can be set up to monitor data quality of incoming data at regular intervals and deliver alerts when an aberration is detected.

Politicians in each country must be transparent in data sharing and mechanism of generating public health information. The process must consensus to rapid law and regulation transformation time to time. Strong top-down leadership and champions of change are essential to move a country for better future in public health management.

\section{Ethics, Equity and Social Considerations}

New advances in technology also have an indirect impact in several social and ethical considerations. Ensuring the distribution of risks and benefits of new advances should equitable and less culture sensitive. Certain technologies will have important implications for societal structures, religious belief, and cultural practice. Innovation potential to use genomeediting technology for enhancement raises concerns about creating worries for individuals, especially those with comorbidities. The effects of such a scenario closely related to public policy in the concern of reduction in the frequency of birth defects. Disabilities will be led to weaker public support for accommodating the needs of people for economy and workforce development. 
Three ethical issues was highlight relating to health information for public health surveillance (Kostkova, P., 2018):

a) Sharing data across various early warning tools to support risk assessment.

b) Legal frameworks for public health data sharing to unlock the potential of populationlevel datasets for research with no impact on citizen's privacy.

c) Strict regulation of the IT industry with regards to manipulating user data.

A study on implementation of information systems conducted among employees in public health sector of developing countries. In the result, the study reported behavioral intention influenced by perceived need, perceived information sharing, and effort expectancy. Meanwhile, perceived awareness had a negative impact behavioral intention (Mukred, A. et al., 2017). Ethical concerns are paramount in the creation and innovation of technologies as the policymakers work to enact new governance frameworks, attention to potential social and culturally sensitive factors. Dialogue session and participation of patients and the public in the process of developing policy should be embedded consistently.

\section{Norms, Standards, Responsible Conduct}

Ethical norms and standards to govern behavior differ across the countries. A scientific community comprising stakeholders of various categories like public and social, ethical, and religious groups requisite to develop norms and standards for implementation of new technologies that distinguish between acceptable and unacceptable behavior. Different society group should play role to consider the implications of new advances in the context of local diverse historical, cultural, and social characteristic. Thus, specific regulatory or legal frameworks will differ from country to country. Ethical principles for emerging technologies should be strictly consider in policy making, especially outlined for human genome editing.

\section{Data Ownership, Privacy and Sharing}

Data ownership, privacy and sharing remain global threat in the world. Patients have a right to own their data and control over it. Regulatory systems and other protocols must be in place to protect patients' rights regarding their data and to ensure that patients feel comfortable sharing their personal health information. Guarding bias is challenging as big data and analytics and AI become more perceived in healthcare. Software and algorithms of AI remain subjective and wide range of grey areas. Computer hackers are extremely searching for this data underground. Selling patient information data to organizations become an unseen market and big scam nowadays.

A systematic review study on the public health informatics data highlighted the issue of healthcare data and ownership in the context of big data is poorly researched. There is lack of consistency and integrity. Subsequently, this has impacted the policy decisions and the necessary legal regulations. The authors urge for future research to investigate on the issue of ownership as a core research question. In interim, researchers need to increase the body of knowledge regarding the development of big data management with adequate policies and relevant legal frameworks in compliance with ethical standards (Mirchev, M. et al., 2020). 


\section{Biosecurity and Biosafety}

Unintentional misuse or deliberate malicious use of research raise concerns in new biotechnologies and scientific advances. National security and biotechnology communities reported that genome editing had become a global danger for the world. Harmful biological agents or products can be used improperly as biological terrorism. Subsequently, infectious might spread around the world and cause devasting health impact to living nature. World Economic Forum also highlighted transformation of biological risk in their reports. Another concern in emerging technologies for new biological threats is manufacturing and releasing of agents into the environment. This not only include healthcare laboratories but also in areas of chemicals, fuels, electronics, and others. In agriculture industry, stakeholders have sufficient knowledgeable of biosecurity risks and had established network connections. However, they expressed a less interest in or unwilling to report a potential biosecurity threat (Curnock, M. et al., 2017). Despites benefits to the world; it is necessary to manage the risks through rigorous transparency and oversight requirements.

Most of the time "Real-time" surveillance is impossible in the systems like the French communicable diseases computer network and the European influenza surveillance scheme only able to collect and analyze data at least once per week. Meanwhile, the QFLU programs have shown capability of daily reporting in the 2006 influenza season. Unfortunately, the system applied depersonalized data collecting method from electronic health records, a data source which is unavailable in most countries (Hitchcock, P. et al., 2007).

\section{Cybersecurity}

According to survey in 2016, healthcare was the fifth most targeted industry by the computer hackers. Nearly 16 million patient's databases were stolen from healthcare organizations related bodies (Why has Healthcare become Such a Target). In future, this threat is only likely to exaggerate with cyberattacks becoming increasingly sophisticated. Hospitals and health systems are vulnerable place to cyberattacks that jeopardy patient information. These issues range from malware that suppress the integrity of systems and privacy of patients and disrupt facilities ability to provide patient care. There is possibility of overtaking infrastructure security, such as the electric grid, could threaten hospitals from functioning and safety. Besides that, medical device linked to a network possess extreme risk from being manipulated and exploited by hackers. In Singapore, nearly 13,000 data of individuals was stolen and published online in 2016. The health authority had apologized and strengthens their block access to confidentiality of the data (Watts, J.M. et al., 2019).

The both parties, health systems and individuals will need to become more aware of their vulnerability to cyberattacks and start taking necessary measures to protect themselves. Policymakers can take vigilant efforts by enacting policies and more stringent control over privacy and data security.

\section{Conclusion}

In the past two decades, public health surveillance has evolved from non-automated to electronic-based surveillance and from infectious diseases to non-infectious conditions. Considering this widened surveillance lens, we must be open to new data sources and methods and preserve the essential systems in place. Given the proliferation of data systems, unique workforce needs, and new tools and technologies, we must be open to a new way of doing 
surveillance. Training and revolutionizing the way epidemiologists and public health professionals get, transfer, and use data when there are limited institutions to provide formal education, specifically in public health informatics, is one of the challenges we need to address. Transforming public health surveillance through open public health information has gained significant attention recently. Community demands on transparency and accessibility of health surveillance have both supporting and opposing opinions from the practitioners. When it is needed, restricted and constrained public health information could delay public health efforts to prevent or control an outbreak as soon as possible. However, ethical, security, and privacy issues must be considered before sharing essential and potentially sensitive public health information, affecting the risk communication process. Guiding principles to navigate the complex privacy, communication, and security are needed to ensure that collaboration and sharing occur in a manner that is ethically and socially just, efficient, and equitable.

Public health surveillance has evolved that it involves different stakeholders form various discipline. This multi-sectoral nature of public health surveillance demands for integration as to reduce unnecessary variation in the conduct of surveillance. By doing this, health surveillance involving various sectors such as those involved in one health approach can be further enhanced to ensure effectiveness and efficiency. However, challenges in the form of limited resources need to be addressed in order to transform this idea into reality. Public private partnership, academic sectors contribution and strengthened collaboration between stakeholders is the only way forward for sustainable development in public health surveillance. It may take time, but the sum of our efforts is greater than the parts.

\section{Conflicts of Interest}

The authors would like to declare there is no conflict of interest.

\section{Acknowledgements}

The authors would like to thank and acknowledge all the lecturers in Public Health Medicine Department, Universiti Malaysia Sabah as well as the bathcmates from DrPH candidates for their continuous support and assistance in making this review possible.

\section{Funding}

This review was self-funded.

\section{References}

45 Fascinating IoT Statistics for 2021, The State of the Industry, [cited 2021 May 26]. https://dataprot.net/statistics/iot-statistics/

ARGUS-IS (Autonomous Real-Time Ground Ubiquitous Surveillance Imaging System). Persistent Surveillance, BAE Systems, United States. https://www.baesystems.com/enus/product/autonomous-realtime-ground-ubiquitous-surveillance-imaging-system-argusis

Aziz, H.A. A Review of the Role of Public Health Informatics in Healthcare. (2016). J. Taibah Univ. Med. Sci., 12(1), 78-81. https://doi.org/10.1016/j.jtumed.2016.08.011

Bahk, C.Y., Scales, D.A., Mekaru, S.R., Brownstein, J.S., Freifeld, C.C. (2015). Comparing Timeliness, Content, and Disease Severity of Formal and Informal Source Outbreak Reporting. BMC Infect. Dis., 15(1), 135 (1-6). https://doi.org/10.1186/s12879-015-0885-0

Baker, M.G., Fidler, D.P. (2006). Global Public Health Surveillance under New International Health 
Regulations. Emerg. Infect. Dis., 12(7), 1058-1065. https://doi.org/10.3201/eid1207.051497

Balajee, S.A., Salyer, S.J., Greene, C.B., Sadek, M., Mounts, A.W. (2021). The Practice of Event based Surveillance: Concept and Methods. Glob. Secur. Heal. Sci. Policy., 6(1), 1-9. https://doi.org/10.1080/23779497.2020.1848444

Bellod, C.J.L., Møller A.F., Lund, O. (2018). Public Health Surveillance using Decentralized Technologies. Blockchain Health Today, 1(1), 1-14. https://doi.org/10.30953/bhty.v1.17

Bhattacharya, S., Singh, A., Hossain, M. (2019). Strengthening Public Health Surveillance through $\begin{array}{lllll}\text { Blockchain } \quad \text { Technology., } & \text { AIMS Public }\end{array}$ https://doi.org/10.3934/publichealth.2019.3.326

Blench, M. (2008). Global Public Health Intelligence Network (GPHIN). AMTA $2008-8^{\text {th }}$ Conf. Assoc. Mach. Transl Am., 299-303. https://aclanthology.org/2008.amta-govandcom.2

Bonilla, A.D.K., Holguin, R.Y., Cortes, B.I., Cardona, T.M.C., García, B.A., Bedoya, A.H.A., (2020). Coronavirus Infections Reported by ProMED, February 2000 - January 2020. Travel Med. Infect. Dis. 35, 101575(1-5). https://doi.org/10.1016/j.tmaid.2020.101575

Brownstein, J.S., Freifeld, C.C. (2008). Evaluation of Internet-Based Informal Surveillance for Global Infectious Disease Intelligence. Int. J. Infect. Dis., 12(S1), e193-e194. https://doi.org/10.1016/j.ijid.2008.05.481

Budd, J., Miller, B.S., Manning, E.M., Lampos, V., Zhuang, M., Edelstein, M., Rees, G., Emery, V.C., Stevens, M.M., Keegan, N., Short, M.J., Pillay, D., Manley, E., Cox, I.J., Heymann, D., Johnson, A.M., McKendry, R.A. (2020). Digital Technologies in the Public-Health Response to COVID19, Nat. Med., 26(8), 1183-1192. http://dx.doi.org/10.1038/s41591-020-1011-4

Butler, D. (2013). When Google Got Flu Wrong, Nature, 494(7436), 155-156. https://doi.org/10.1038/494155a

Butler, D. (2017). World's Foremost Institute on Death and Disease Metrics Gets Massive Cash Boost. Nature, 542(7639), 1. https://doi.org/10.1038/nature.2017.21373

CDC (Centers for Disease Control and Prevention). (2012). Principles of Epidemiology: Lesson 5, Appendix E, Appendix E. Limitations of Notifiable Disease Surveillance and Recommendations for Improvement, Self-Study Course SS1978. https://www.cdc.gov/csels/dsepd/ss1978/lesson5/appendixe.html

CDC (Centers for Disease Control and Prevention). (2014). Public Health 101 Series: Introduction to Public Health Surveillance. Atlanta, GA: U.S. Department of Health and Human Services. https://www.cdc.gov/training/publichealth101/surveillance.html

Choi B.C.K. (2012). The Past, Present, and Future of Public Health Surveillance. Scientifica (Cairo), 2012, 875253 (1-26). https://doi.org/10.6064/2012/875253

Connelly, D.P., Park, Y.T., Du, J., Theera, A.N., Gordon, B.D., Bershow, B.A., Gensinger, R.A.Jr., Shrift, M., Routhe, D.T., Speedie, S.M. (2012). The Impact of Electronic Health Records on Care of Heart Failure Patients in the Emergency Room. J. Am. Med. Inform. Assoc., 19(3), 334340. https://doi.org/10.1136/amiajnl-2011-000271

Curnock, M., Farbotko, C., Collins, K., Robinson, C.J., Maclean, K. (2017). Engaging with Risk (or not): Shared Responsibility for Biosecurity Surveillance and the Role of Community Gardens. Geogr. Res., 55(4), 379-394. https://doi.org/10.1111/1745-5871.12231

Davis, E. (2017). The Problems of Big Data and What to do About Them. World Economic Forum. https://www.weforum.org/agenda/2017/02/big-data-how-we-can-manage-the-risks

Flu \& Ebola Map, Virus \& Contagious Disease Surveillance [cited 2021 May 25]. https://healthmap.org/en/

Friedrich, M.J. (2019). WHO's Top Health Threats for 2019. JAMA, 321(11), 1041 (1-15). https://doi.org/10.1001/jama.2019.1934

Ginsberg, J., Mohebbi, M.H., Patel, R.S., Brammer, L., Smolinski, M.S., Brilliant, L. (2009). Detecting Influenza Epidemics Using Search Engine Query Data. Nature., 457(7232), 1012- 


\section{4. https://doi.org/10.1038/nature07634}

Health Management. Radiology Management, ICU Management, Healthcare IT, Cardiology Management, Executive Management. https://healthmanagement.org/c/hospital/event/ehma2020-annual-conference-european-health-management-association

Herwehe, J., Wilbright, W., Abrams, A., Bergson, S., Foxhood, J., Kaiser, M., Smith, L., Xiao, K., Zapata, A., Magnus, M. (2012). Implementation of An Innovative, Integrated Electronic Medical Record (EMR) and Public Health Information Exchange for HIV/AIDS. J Am. Med. Inform. Assoc., 19(3), 448-452. https://doi.org/10.1136/amiajnl-2011-000412

Hitchcock, P., Chamberlain, A., Van, W.M., Inglesby, T.V., O’Toole, T. (2007). Challenges to Global Surveillance and Response to Infectious Disease Outbreaks of International Importance. Biosecur. Bioterror., 5(3), 206-227. https://doi.org/10.1089/bsp.2007.0041

How IoT, AI, and blockchain will revolutionize business. (2018). Transformational Technologies: Today, Oracle. https://www.oracle.com/a/ocom/docs/transformational-tech-wp.pdf

How One Man Saved his Country from A Nightmare Virus Called Nipah: Goats and Soda: NPR. [cited 2021 May 26]. https://www.npr.org/sections/goatsandsoda/2017/02/25/515258818/a-tastefor-pork-helped-a-deadly-virus-jump-to-humans

Huang, J., Loschen, W. (2019). Potential Applications of Emerging Technologies in Disease Surveillance. Online J. Public Health Inform., 11(1), e340 (1-2). https://doi.org/10.5210/ojphi.v11i1.9821

Jayatilleke, K. (2020). Challenges in Implementing Surveillance Tools of High-Income Countries (HICs) in Low Middle Income Countries (LMICs). Curr. Treat. Options Infect. Dis., 12(3), 191201. https://doi.org/10.1007/s40506-020-00229-2

Johns Hopkins Team Launches Temperature-Tracking Study and App to Map and Monitor Potential COVID-19 Cases [cited 2021 May 26]. https://hub.jhu.edu/2020/04/30/johns-hopkins-covidtemperature-tracking-app/

Joshi, A., Perin, D.M.P. (2012). Gaps in the Existing Public Health Informatics Training Programs: A Challenge to the Development of A Skilled Global Workforce. Perspect. Health Inf. Manag., 9, 1-13. https://www.ncbi.nlm.nih.gov/pmc/articles/PMC3510646/pdf/phim0009-0001c.pdf

Kamradt, S.A. (2019). The International Health Regulations (2005), International Organizations Law Review, 16(2), 242-271. https://doi.org/10.1163/15723747-01602002

Kim, G.U., Kim, M.J., Ra, S.H., Lee, J., Bae, S., Jung J, Kim., S.H. (2020). Clinical Characteristics of Asymptomatic and Symptomatic Patients with Mild COVID-19. Clin. Microbiol. Infect., 26(7), 948.e1-948.e3. https://doi.org/10.1016/j.cmi.2020.04.040

Kirkwood A, Guenther E, Fleischauer AT, Gunn J, Hutwagner L, Barry MA. (2007). Direct Cost Associated with the Development and Implementation of A Local Syndromic Surveillance System. J. Public Heal. Manag. Pract., 13(2), 194-199. https://doi.org/10.1097/00124784200703000-00017

Klembczyk, J.J., Jalalpour, M., Levin, S., Washington, R.E., Pines, J.M., Rothman, R.E., Dugas, A.F. (2016). Google Flu Trends Spatial Variability Validated Against Emergency Department Influenza Related Visits. J. Med. Internet Res. 18(6), e175(1-11). https://doi.org/10.2196/jmir.5585

Kostkova, P. (2018). Disease Surveillance Data Sharing for Public Health: The Next Ethical Frontiers. Life Sci. Soc. Policy., 14(1), 16(1-5). https://doi.org/10.1186/s40504-018-0078-x

Kumar, A., Sharma, K., Singh, H., Naugriya, S.G., Gill, S.S., Buyya, R. (2021). A Drone-Based Networked System and Methods for Combating Coronavirus Disease (COVID-19) Pandemic. Future Gener. Comput. Syst., 115, 1-19. https://doi.org/10.1016/j.future.2020.08.046

Lampos, V., Miller, A.C., Crossan, S., Stefansen, C. (2015). Advances in Nowcasting Influenza-Like Illness Rates Using Search Query Logs. Sci Rep., 5, 12760(1-10). https://doi.org/10.1038/srep12760 
Magid, A., Gesser, E.A., Green, M.S. (2018) The Role of Informal Digital Surveillance Systems before, During and After Infectious Disease Outbreaks: A Critical Analysis. p. 189-201. Radosavljevic V., Banjari I., Belojevic G. (Eds.) In: Defence Against Bioterrorism. NATO Science for Peace and Security Series A: Chemistry and Biology. Springer, Dordrecht. https://doi.org/10.1007/978-94-024-1263-5_14

Mandyata, C.B., Olowski, L.K., Mutale, W. (2017). Challenges of Implementing the Integrated Disease Surveillance and Response Strategy in Zambia: A Health Worker Perspective. BMC Public Health., 17(1), 746(1-12). https://doi.org/10.1186/s12889-017-4791-9

Mathers, C.D., Fat, D.M., Inoue, M., Rao, C., Lopez, A.D. (2005). Counting the Dead and What they Died From: An Assessment of the Global Status of Cause of Death Data. Bull. World Health Organ., 83(3), 171-177. https://apps.who.int/iris/handle/10665/269355

Mccarthy, J. What is Artificial Intelligence? 2007, 1-14. http://wwwformal.stanford.edu/jmc/whatisai/

McNabb, S.J.N., Tyland, P., Sylvester, J., Shaikh. A. (2017). Informatics Enables Public Health Surveillance. J. Heal. Spec., 5(2), 55-59. http://dx.doi.org/10.4103/jhs.JHS 2817

Meng, H., Liao, Q., Suen, L.K.P., O’Donoghue, M., Wong, C.M., Yang, L. (2016). Healthcare Seeking Behavior of Patients with Influenza like Illness: Comparison of the Summer and Winter Influenza Epidemics. BMC Infect. Dis. 16(1), 499 (1-9). https://doi.org/10.1186/s12879-016$\underline{1821-7}$

Mirchev, M., Mircheva, I., Kerekovska, A. (2020). The Academic Viewpoint on Patient Data Ownership in the Context of Big Data: Scoping Review. J. Med. Internet Res., 22(8), e22214 (120). https://doi.org/10.2196/22214

Mukhtar, H., Rubaiee, S., Krichen, M., Alroobaea, R. (2021). An IoT Framework for Screening of COVID-19 Using Real-Time Data from Wearable Sensors. Int. J. Environ. Res. Public Health., 18(8), 4022(1-17). https://doi.org/10.3390/ijerph18084022

Mukred, A., Singh, D., Safie, N. (2017). Investigating the Impact of Information Culture on the Adoption of Information System in Public Health Sector of Developing Countries. Int. J. Bus. Inf. Syst., 24(3), 261-284. https://doi.org/10.1504/IJBIS.2017.082036

PHII (Public Health Informatics Institute). (2020). Define Public Health Informatics. Decatur, Georgia. https://phii.org/how-we-do-it/defining-public-health-informatics/

Pollett, S., Boscardin, W.J., Azziz, B.E., Tinoco, Y.O., Soto, G., Romero, C., Kok, J., Biggerstaff, M., Viboud, C., Rutherford, G.W. (2017). Evaluating Google Flu Trends in Latin America: Important Lessons for the Next Phase of Digital Disease Detection. Clin. Infect. Dis., 64(1), 3441. https://doi.org/10.1093/cid/ciw657

ProMED - ProMED-mail. [cited 2021 May 25]. https://promedmail.org/

Rama K.S., Lavanyah, S.A.A.R. (2021). Descriptive Epidemiology of the First Wave of COVID-19 in Petaling District, Malaysia: Focus on Asymptomatic Transmission. Western Pacific Surveill. Response J., 12(2), 82-88. https://doi.org/10.5365/wpsar.2020.11.4.001

Singapore's COVID-19 Temperature Scans and Tracking Leading the Way. [cited 2021 May 26]. https://thenewdaily.com.au/news/national/2020/03/19/singapore-coronavirus-temperature-scans/

Watts, J.M., Venkat, P.R. HIV Status of More Than 14,000 People Leaked in Singapore Data Breach WSJ. The Wall Street Journal. 2019. https://www.wsj.com/articles/hiv-status-of-more-than-14000-people-leaked-in-singapore-data-breach-11548679969

What is IoT? Defining the Internet of Things (IoT), Aeris, [cited 2021 May 26]. https://www.aeris.com/in/what-is-iot/

Whitelaw, S., Mamas, M.A., Topol, E., Van, S.H.G.C. (2020). Applications of Digital Technology in COVID-19 Pandemic Planning and Response. Lancet Digit. Heal., 2(8), e435-E440. http://dx.doi.org/10.1016/S2589-7500(20)30142-4 
WHO (World Health Organization). (2020). Public Health Surveillance for COVID-19: Interim Guidance. https://www.who.int/publications/i/item/who-2019-nCoV-surveillanceguidance2020.8

WHO Coronavirus (COVID-19) Dashboard with Vaccination Data, [cited 2021 May 24]. https://covid19.who.int/

WHO, Epidemic Intelligence - Systematic Event Detection. (2020). [cited 2021 May 24]. http://www.who.int/csr/alertresponse/epidemicintelligence/en/

Why has Healthcare become Such a Target for Cyber-Attackers? https://theconversation.com/why-hashealthcare-become-such-a-target-for-cyber-attackers-80656

Wright, W.F., MacKowiak, P.A. (2021). Why Temperature Screening for Coronavirus Disease 2019 with Noncontact Infrared Thermometers Does Not Work. Open Forum Infect Dis., 8(1), ofaa603(1-3). https://doi.org/10.1093/ofid/ofaa603

Zeng, D., Cao, Z., Neill, D.B. (2021). Chapter 22: Artificial Intelligence Enabled Public Health Surveillance from Local Detection to Global Epidemic Monitoring and Control. p. 437-453. In: Artificial Intelligence in Medicine, Lei, X., Maryellen, L.G., James, K.M. (Eds), Academic Press. http://dx.doi.org/10.1016/B978-0-12-821259-2.00022-3 